Empoderamiento y autonomía de las mujeres en la investigación sociodemográfica actual

\title{
Brígida García*
}

En este artículo se analizan los conceptos de empoderamiento y autonomía de las mujeres en un esfuerzo por contribuir a precisar el significado de cada uno y su importancia, asi como avanzar en su estudio y medición. En primer lugar abordamos los antecedentes del término empoderamiento y las dimensiones de la realidad que han sido tomadas en cuenta en su definición; también establecemos las diferencias entre empoderamiento y autonomía. En un segundo momento hacemos hincapié en los aspectos metodológicos y analizamos los esfuerzos de distintas especialistas y organismos internacionales por diseñar indicadores en esle campo de interés. En este último contexto recibe atención particular el papel de los indicadores directos de empoderamiento $y$ autonomía basados en información de encuestas probabilísticas.

Palabras clave: empoderamiento, autonomía, estatus, posición, condición y situación de las mujeres.

Fecha de recepción: 9 de diciembre de 2002.

Fecha de aceptación: 17 de enero de 2003.

\section{Introducción}

El empoderamiento y la autonomía de las mujeres están hoy presentes en los esfuerzos y en el lenguaje de muy diferentes actores sociales (activistas, académicos/as, funcionarios/as gubernamentales y de organismos internacionales). No obstante, hay que reconocer que en muchos casos estos términos permanecen ambiguos y faltos de precisión, y que en México y América Latina no han sido plenamente incorporados en la investigación social y poblacional. En este trabajo buscamos sumarnos a diversos esfuerzos actuales por esclarecer el significado de dichos conceptos y dar un paso adelante en su estudio y medición concreta (véase León, 1997a; Presser y Sen, 2000a y 2000b).

Las secciones que siguen centran su análisis en los antecedentes, el alcance, y las distintas dimensiones involucradas en el empoderamiento y (o) la autonomia de las mujeres, así como en las diferencias

* Profesora-investigadora del Centro de Estudios Demográficos y de Desarrollo Urbano de El Colegio de México. Correo electrónico: bgarcia@colmex.mx 
entre un concepto y otro. Posteriormente se tratan los problemas metodológicos respondiendo a un particular interés por explicitar las posibles maneras de acercarse al empoderamiento (y a las transformaciones relacionadas, como la autonomía femenina) mediante diferentes tipos y técnicas de investigación, con un énfasis especial en las encuestas, que son parte relevante del quehacer sociodemográfico. Recibe atención particular el diseño de indicadores sobre los cambios en la vida de las mujeres objeto de análisis. Por último se sintetizan los principales ejes del debate conceptual y metodológico, y se procura destacar la importancia del estudio del empoderamiento y la autonomía para comprender la condición femenina y las transformaciones poblacionales.

Antes de proseguir con el desarrollo de este trabajo es preciso tomar posición en este apartado introductorio sobre el el empleo en nuestro idioma de los términos empoderamiento y autonomía. En el caso de autonomía se trata de una palabra con raíces conocidas y de larga trayectoria en la lengua española. Según el Diccionario de la Real Academia Española (DRAE), la autonomía es la condición de la persona que no depende de nadie, en ciertos aspectos, y cuando se trata de estados o pueblos, la autonomía es el gozo de la entera independencia política. El uso del término empoderamiento, en cambio, ha dado pie a muchos debates, y no existe en este momento una posición unánime al respecto. Si bien en muchas ocasiones el término original en inglés (empowermenl) ha sido traducido como habilitación y también como potenciación (ésta ha sido, por ejemplo, la manera de proceder en los documentos oficiales de las Naciones Unidas), desde nuestra perspectiva tales aproximaciones, aunque buenas, no logran rescatar los distintos matices del término en inglés. ${ }^{1}$

Como se verá en la próxima sección, el empowerment tiene que ver cọn la ampliación de las capacidades individuales, pero también con el acceso a las fuentes de poder. De esta suerte, el que el término en inglés contenga la palabra power ha sido considerado uno de sus rasgos más sobresalientes (véase Batliwala, 1994). Partiendo de esta premisa,

1 Según el Webster's International Dictionary, empower en inglés tiene dos principales acepciones: 1) to give oficial authority $t o$, delegate legal power $t o$, commissiom, authorize, y 2) to give faculties or abilities to, enable. En el Oxford English Dictionary la entrada empower fue bastante tardía, porque aparece apenas en textos de la segunda mitad del siglo XVII; dice que es "to invest legally or formally with power or authority, to authorize, to licence" (Venier, 1996: 17). 
existiría la opción de emplear en español "apoderamiento", que especialistas como Martha Elena Venier (1996) consideran más afín en nuestra lengua (el verbo sería "apoderar", pero también recomienda el uso de la perífrasis "dar poder a", según el contexto de que se trate). Apoyada en varios textos clásicos y en una exploración de distintos diccionarios, Venier considera que el verbo "apoderar" es una buena opción, pues se trata de un verbo de uso antiguo y con un buen número de variantes, e incluye tanto la acepción de "hacer poderoso" como la de "hacerse poderoso". En cambio no recomienda empoderar porque aunque reconoce que este verbo existe en varios diccionarios que lo presentan como un término en desuso y sinónimo de apoderary también de poderio, no cuenta con testimonios de autoridades (Venier, 1996).

Empoderarsí existe entonces en español, pero no tiene antecedentes claros y su uso no es recomendado por los lingüistas. Sin embargo, a pesar de su dudosa procedencia, gradualmente se ha ido imponiendo dentro del movimiento de mujeres en América Latina, así como en el lenguaje de funcionarios gubernamentales nacionales e internacionales interesados en los temas de desarrollo, pobreza y organizaciones populares. En este texto nos sumamos a la elección que se ha hecho en estos diferentes campos del quehacer social, atendiendo principalmente a las razones que expone de manera convincente Magdalena León en su libro sobre Poder y empoderamiento de las mujeres (León, 1997a). Esta autora explica que privilegia el uso de empoderar y empoderamiento porque estos términos -a diferencia de otros- denotan acción y surgen en el contexto de movimientos sociales que buscan la transformación de las condiciones de subordinación y explotación. ${ }^{2}$ Asimismo considera que trasmiten de manera más efectiva la idea de que el sujeto se convierte en agente activo de su proceso de cambio. Defiende asimismo la posición de que mediante su uso se puede contribuir "a impulsar cambios en la cultura, en particular en los imaginarios sociales sobre la relación de la mujer en el poder" (León, 1997: 6).

2 "El prefijo en (em en bilabiales) es herencia del latín in [...] que en las lenguas romances, lo mismo que en inglés [...] por lo regular denota acción, y a veces suele aumentar o variar el significado..." (Venier, 1996: 18). 


\section{Antecedentes}

El surgimiento del concepto de empoderamiento está estrechamente ligado a la acción de diferentes tipos de movimientos sociales (de derechos civiles, de mujeres) que pretenden cambiar las relaciones y la distribución del poder establecidas. ${ }^{3}$ En el campo de las políticas y los estudios de población, este concepto ganó plenamente legitimidad en el marco de la Conferencia Internacional sobre Población y Desarrollo (CIPD) de 1994, cuyo programa de acción dedicó un capítulo especial a la igualdad, la equidad y el empoderamiento de las mujeres. Se trató de la primera gran conferencia internacional de población convocada por las Naciones Unidas donde las mujeres activistas a favor de la salud femenina desempeñaron un papel preponderante (véase Presser, 1997; Sen y Batliwala, 2000).

Para entender a cabalidad la relevancia del concepto de empoderamiento en el caso de las mujeres, consideramos muy útil la referencia a los trabajos de Batliwala (1994) y de León (1997b), donde se hacen recuentos de los desarrollos teóricos clave y de los debates y acciones dentro del movimiento de mujeres que abonaron el terreno para el surgimiento del mismo. En particular se refieren a las críticas feministas que denostaban las estrategias de desarrollo puestas en marcha durante los setenta porque no tomaban en cuenta los factores estructurales que perpetuaban la subordinación femenina. A partir de estas críticas surgió la necesidad de distinguir entre la condición y posición de las mujeres y entre sus intereses prácticos y estratégicos (véase Molyneaux, 1985; Young, 1988; Moser, 1989). Según Kate Young la condición de las mujeres hace referencia a su estado material (salarios bajos, mala nutrición, carencias en lo que respecta a la salud, educación y capacitación), mientras que su posición indica su estatus económico y social en comparación con el de los hombres. Por su parte, Maxine Molyneaux considera que hay que diferenciar entre los intereses prácticos de las mujeres (alimentación, salud, agua, combustible, cuidado de los hijos, educación) y sus metas estratégicas tendentes a cambiar su

${ }^{3}$ Varias autoras aseguran que el concepto de empoderamiento tiene su origen en los movimientos a favor de los derechos civiles de los afronorteamericanos en Estados Unidos en los años sesenta. Ha constituido una herramienta de análisis y acción central en el movimiento feminista desde los setenta, y se utiliza en diferentes campos disciplinarios como la educación, el derecho, la ciencia política, la psicología, la antropologia, la economia, los estudios sobre las mujeres y el género y la sociodemografia (sobre los orígenes del concepto, véase León, 1997b; Stromquist, 1997). 
subordinación. En este contexto surge el concepto de empoderamiento como una herramienta clave para alcanzar los intereses estratégicos y así cambiar la posición de las mujeres. Se trata de una manera diferente de percibir el desarrollo, viéndolo no desde arriba, sino desde las bases. En el caso del movimiento de mujeres fue articulado por primera vez por Gita Sen y Karen Grown en el marco de los trabajos de DAWN $^{4}$ y en un documento preparado para la Tercera Conferencia Mundial de la Mujer que tuvo lugar en Nairobi en 1985 (véase Sen y Grown, 1987; Batliwala, 1994; León, 1997b).

\section{Aspectos conceptuales del empoderamiento}

A la luz de los antecedentes mencionados destaca la importancia de asociar el término empoderamiento al desafio de las fuentes de poder, a la lucha por cambiar las relaciones de subordinación femenina que existen hoy día, y a la habilidad para definir el curso a seguir. Si bien gran parte de los (las) especialistas estarían de acuerdo con este planteamiento general, no todas/os priorizarían de la misma manera los aspectos que intervienen en este proceso, o los ámbitos sociales, organizacionales o personales que deben tomarse en cuenta. Algunos hacen hincapié en la potenciación de las capacidades individuales o empresariales, en la generación de la autoconfianza, pero esta posición ha sido criticada -desde nuestra perspectiva con razón- por aquellos que consideran que si se reduce el proceso de empoderamiento a estas dimensiones se puede caer en el individualismo, restando importancia a la cooperación y minimizando la necesidad de modificar las relaciones de género y la estructura social existentes (véase Riger, 1997; Young, 1997). ${ }^{5}$ Por esto una de las definiciones más aceptadas y utilizadas de empoderamiento se refiere más bien al logro del control en muy diferentes ámbitos: el de los recursos (humanos, físicos, intelectuales, financieros) y el de la ideología (creencias, valores, y actitudes):

${ }^{4}$ DAWN (Development Alternatives with Women for a New Era) O MUDAR (Alternativas de Desarrollo con Mujeres para una Nueva Era) en español, es una red de estudiosas feministas y activistas de paises en desarrollo que se constituyó en la India en 1984.

${ }^{5}$ Por esta razón respaldamos la idea de que la traducción al español de empowerment como potenciación no rescata plenamente los distintos matices presentes en el têrmino original en inglés. 
Los bienes materiales sobre los cuales puede ejercerse el control pueden ser físicos, humanos o financieros, tales como la tierra, el agua, los bosques, los cuerpos de las personas, el trabajo, el dinero, y el acceso a éste. Los recursos intelectuales incluyen los conocimientos, la información y las ideas. El control sobre la ideología significa la habilidad para generar, propagar, sostener e institucionalizar conjuntos específicos de creencias, valores, actitudes y comportamientos, determinando virtualmente la forma en que las personas perciben y funcionan en un entorno socioeconómico y político dado (Batliwala, 1997: 192).

En relación con lo anterior suele discutirse si el empoderamiento es un proceso intrínseco o extrínseco, si puede ser inducido desde afuera o se trata de una transformación interior. La posición que desde nuestro punto de vista tiene más consenso es que ambos aspectos son relevantes y no se deben tratar como opuestos, pero algunas autoras sostienen que es necesaria una modificación interna para que el cambio pueda perdurar, mientras que otras le dedican más atención al papel de la colectividad, a las organizaciones de base y a los agentes catalizadores del proceso de empoderamiento (veáse Riger, 1997; Young, 1997; León, 1997b; Sen, 1998).

¿Cómo se comenzaría y procedería el proceso de empoderamiento, y qué tipos de esfuerzos se han hecho para precisar sus facetas, componentes o dimensiones? Muchos/as coinciden en que no se trata de un proceso lineal, de ahí que a veces se haya planteado más bien la idea de que avanzaría como una espiral o de que se puede lograr el empoderamiento en un ámbito como la familia, pero no en la comunidad (UNICEF, 1997; Sen y Batliwala, 2000). Resulta en todos los casos esencial la concientización, la cual según Paulo Freire se refiere al desarrollo de una conciencia crítica, reflexiva, que promueve la creatividad y la capacidad de tomar decisiones y transformar la realidad (Freire, 1973). Este legado de Freire es reconocido por gran parte de las autoras especialistas en el tema del empoderamiento. Según Batliwala (1994), Ias educadoras populares feministas se apoyaron en los trabajos de Freire -que por cierto no hizo hincapié en las desigualdades de género- pero avanzaron más allá de la concientización y procuraron la organización de los pobres para luchar por el cambio (el cual involucraría el control de los recursos y el de la ideología, como vimos arriba).

Por su parte, autoras como Stromquist (1997) distinguen entre los componentes cognitivos (comprensión de la situación de subordinación), los psicológicos (desarrollo de la autoestima y la confianza), los económicos (acceso a actividades productivas que proporcionen 
por lo menos algún grado de independencia financiera), y los políticos (habilidad para organizar y movilizar cambios sociales). Finalmente, Rowlands (1997) ha planteado un modelo de empoderamiento con tres dimensiones principales: la personal (desarrollo del sentido de ser, de la confianza y de la capacidad individual), la referente a las relaciones cercanas (habilidad para negociar la naturaleza de la relación y las decisiones que se toman en su interior), y la colectiva (participación en estructuras políticas locales o informales, internacionales o formales; acción fundamentada en un modelo cooperativo y no competitivo).

El analizar la naturaleza del poder y el diferenciar entre los tipos de poder existentes también forman parte importante de la comprensión del proceso de empoderamiento, sus alcances y sus fines. La influencia de autores como Gramsci (1971), Foucault $(1972,1980)$ y Lukes $(1974,1986)$ es clara en varios de los trabajos más conocidos sobre el tema. En este contexto vale la pena indicar que el poder no sólo es algo que se tiene, sino más bien una relación social que determina el acceso y el control de diferentes tipos de recursos; está presente en todos los niveles de la sociedad, y los individuos participan en esta compleja red como actores y como objetos, pero al mismo tiempo configuran las esferas de poder (Foucault, 1972, 1980; Wieringa, 1997; Bookman y Morgen, 1988). En lo que concierne a los diferentes tipos de poder, aquel que más se conoce es el poder sobre, el cual puede ser ejercido y estar presente de diversas maneras. Se trata de lograr que otras personas actúen como se desea en conflictos observables, pero también de suprimir los conflictos y de tener la capacidad de decidir sobre qué se decide. Asimismo es posible que una manifestación del poder sobre sea que ni los dominantes ni los dominados estén conscientes de la opresión -por ser ésta estructural y por estar pautada por patrones culturales y prácticas institucionales- y que por tanto no sea fácil ofrecer alternativas del curso a seguir (Lukes, 1974, 1986; Kabeer, 1994; León, 1997b; Rowlands, 1997; Wieringa, 1997).

El poder sobre es generalmente de suma cero y muchas veces conlleva aspectos destructivos. Según León (1997b), esto llevó a que las feministas se negaran hasta hace poco a discutir sobre el poder. No obstante, esta situación ha cambiado notablemente, y con el concepto de empoderamiento han surgido otras maneras de concebir el poder que serían positivas: poder para, poder creativo y facilitador que al no basarse necesariamente en acciones de dominación, en cambio estimula la actividad y abre nuevas posibilidades; poder con, poder que multiplica los 
esfuerzos individuales y que es capaz de demostrar que el todo es mayor que la suma de las partes, y poder desde dentro o poder del interior, que es la habilidad para darle un sentido de control a la propia vida, de resistir el poder de otros y de sentar las bases sobre las cuales es posible construir alternativas (Kabeer, 1994; Rowlands, 1997).

\section{Sobre los conceptos de empoderamiento y autonomía}

¿Qué diferencias podemos encontrar entre los conceptos de empoderamiento y autonomia? Para intentar responder esta pregunta hay que partir del reconocimiento de que muchos especialistas -y los propios documentos de Naciones Unidas- utilizan estos dos términos de manera intercambiable o simultánea; sin embargo, consideramos más apropiada la posición de aquellos que establecen diferencias. Habría que analizar ahora de qué manera se justifican tales diferencias.

Por ejemplo, Nelly Stromquist (1997) argumenta que la autonomía podría muy bien ser una faceta del empoderamiento, y de manera más específica se asemejaría a lo que ella define como la faceta psicológica (otras facetas planteadas por esta autora son la cognitiva, la económica y la política, todas definidas desde una perspectiva individual). La autonomía sería similar al poder desde dentro, que Stromquist considera necesario tener antes de lograr ejercer otros tipos de poder. Esta posición es explícita, pero desde mi perspectiva no queda claro por qué la autonomía, esto es, la capacidad de actuar con independencia y según intereses propios, tendría que restringirse a esta faceta psicológica y no constituir un aspecto de las demás dimensiones que esta autora tiene en cuenta. Asimismo, la idea de que la autonomía -entendida como poder desde dentro- debe preceder a los otros ejercicios del poder es cuestionable, pues se sabe que muchas veces las mujeres actúan con independenciạ en las esferas económica y política, y no necesariamente han conquistado la autonomía en sus relaciones más cercanas.

Tampoco está exento de problemas el planteamiento de Casique (2001a) respecto a que el poder y la autonomía de las mujeres tendrían que ver con dos aspectos distintos de la vida cotidiana: el poder con la participación efectiva en la toma de decisiones, y la autonomía con la actuación por iniciativa propia (libertad de movimiento) y fuera de la influencia de otros. Esta separación puede resultar un tanto artificial y no queda claro por qué se llama a un aspecto podery a otro autonomía cuando ambos constituyen elementos analíticos de un mismo proce- 
so de autoafirmación y control. En un trabajo posterior, Irene Casique avanza en esta dirección al plantear que ambos, el poder en la toma de decisiones y la autonomía, forman parte del proceso de empoderamiento de la mujer (véase Casique, 2001b).

Marina Ariza y Orlandina de Oliveira perciben también que la autonomia y el empoderamiento están analíticamente separados, pero más bien en lo que respecta a la esfera de la realidad en la cual operarían estas transformaciones. Limitan el uso del concepto de empoderamiento a los ámbitos político y social, en comparación con la autonomía y la individuación que las conciben como parte de un proyecto de desarrollo personal. No obstante, estas autoras esbozan la relación que existiría entre ambos aspectos, porque indican que para lograr mayor igualdad, autonomia e individuación, se requiere que las mujeres tengan la capacidad de organizarse y luchar en forma colectiva, ejerciendo un mayor poder político en la comunidad y en la sociedad en general. A esa organización y lucha en el nivel de lo político la denominan "el proceso de empoderamiento" (Ariza y Oliveira, 1996: 41; véase también López y Salles, 1996). Es sugerente el tipo de relaciones que establecen Ariza y Oliveira entre la organización y lucha en el nivel de lo político y la autonomía e individuación en el nivel personal, pero nos parece restrictivo reservar el término empoderamiento sólo para el primer nivel de análisis. Como vimos en la parte conceptual, existe también una perspectiva individual en el desafío de las fuentes de poder y en el eventual control de los diferentes tipos de recursos.

Finalmente, Shireen Jejeebhoy analiza varias definiciones de empoderamiento y autonomía, y considera que convergen en lo que respecta al objetivo que se persigue, esto es, el ganar control sobre la propia vida en lo que toca a la familia, la comunidad, la sociedad y los mercados. Sin embargo, para ella el concepto de autonomía tiene un carácter estático frente al más dinámico del empoderamiento. Por lo tanto estima más apropiado utilizar en su investigación -que se basa en una amplia encuesta en un momento en el tiempo- el concepto de autonomia, entendida como la medida en la cual las mujeres ejercen control sobre sus vidas dentro de sus familias en un momento determinado (Jejeebhoy, 2000: 205). Ésta es una propuesta que responde a un interés de adecuación teórico-metodológica poco común en este campo, sin embargo consideramos que lleva a limitar en demasía el concepto de autonomia restringiéndolo al control que se ejerce en la vida personal y familiar en un momento específico. 
A pesar de los problemas expuestos, varias de las autoras mencionadas apuntan de manera implícita o explícita a una idea que podría desarrollarse en forma más amplia, a saber, que existen relaciones de eslabonamiento o convergencia entre el empoderamiento y la autonomía. Magdalena León también se acerca a esta noción cuando afirma que el empoderamiento conduce a la autonomía personal (León, 1997b). Este tipo de relaciones podría plantearse de manera general, sin restringirlas a un ámbito de la realidad y sin identificar a un fenómeno como dinámico y a otro como estático. Podríamos decir que el cuestionamiento del poder y la búsqueda del control de los diferentes tipos de recursos -ya sea en el plano individual o en el social- llevaría a la independencia y a la actuación según los propios intereses a lo largo del tiempo. O vistas las cosas de otra manera, la autonomia individual (o grupal) sería una de las manifestaciones concretas del empoderamiento individual o colectivo, y podría transformarse en sucesivos momentos. Nos parece que un planteamiento de este tipo ofrece pautas más claras y concretas para la investigación social sobre el tema, y a ello nos abocamos a continuación.

\section{Aspectos metodológicos generales: el diseño de indicadores}

La investigación que se propone conocer el empoderamiento y sus factores asociados se enfrenta a varios desafíos derivados de la complejidad inherente a este proceso. Vimos ya que el empoderamiento es dinámico, multidimensional y no lineal; además, no se restringe a la acción y al cambio individual, sino que tiene que ver con las relaciones interpersonales en muy diferentes ámbitos, y con las transformaciones institucionales y culturales. El hecho de que el proceso de $\mathrm{em}$ poderamiento no se limite a la acción frente a conflictos observables representa un reto a la investigación concreta, pues habría que documentar también los conflictos suprimidos. Finalmente, interesaría dar cuenta tanto de los agentes catalizadores como de los resultados grupales e individuales en torno al control de las fuentes de poder.

Esta gama de elementos apunta claramente a la necesidad de emprender una investigación interdisciplinaria de diversos tipos (cuantitativa y cualitativa), así como a la de combinar distintos niveles y unidades de análisis (individuos de diversas edades y sectores sociales, familias, grupos, comunidades y líderes), y a la importancia de recurrir a diferentes herramientas analíticas (observación participante, 
censos y encuestas, grupos focales, entrevistas en profundidad). Sin embargo hay que tener presente que no siempre se está en posibilidad de efectuar esta ambiciosa y exhaustiva combinación, o no se cuenta con la experiencia o el tiempo necesarios para llevarla a cabo. De esta suerte resulta útil centrar nuestra discusión metodológica en los alcances y limitaciones de los distintos indicadores, así como en las fortalezas y debilidades que presentan en este caso las diversas técnicas de recolección de información.

Se han realizado en los últimos años importantes esfuerzos en torno al diseño de indicadores sobre las desigualdades de género o sobre la condición de la mujer, los cuales no necesariamente descansan en el concepto de empoderamiento o sólo lo utilizan de manera secundaria. ${ }^{6}$ Rebasa claramente los objetivos de este trabajo analizar este rico conjunto de trabajos, por lo que nos centraremos solamente en aquellas propuestas explícitas sobre indicadores de empoderamiento o de autonomía, sin olvidar las diferencias que pretendemos establecer entre ambos aspectos.

Uno de los esfuerzos más conocidos en torno a los indicadores de empoderamiento es el del Programa de las Naciones Unidas para el Desarrollo (PNUD), que a mediados de los noventa publicó por primera vez información para más de 100 países sobre el índice de desarrollo relacionado con la mujer (IDM) y el índice de potenciación de la mujer (IPM) (recordemos que potenciación es la traducción que ha privilegiado Naciones Unidas del término empowerment en inglés).

El IPM -el cual es el índice que ahora más interesa- incorpora información sobre la representación femenina en los parlamentos, en cargos directivos y profesionales, en la fuerza laboral y en el ingreso nacional, intentando de esta manera abarcar la participación de las mujeres en la vida política, económica y social. Se trata de un gran paso hacia la legitimación de posibles indicadores de empoderamiento en una escala internacional, y también de un significativo esfuerzo de recopilación y síntesis de información para gran cantidad de países. Sin embargo, a primera vista parecen muy restringidas las dimensiones específicas incorporadas en este índice. Autoras como Saskia Wieringa (1997) han dejado claro que en el diseño del IPM se ha privilegiado la participación femenina en la vida económica, política y

${ }^{6}$ En el caso de México contamos con varios intentos relevantes en esta dirección (por ejemplo, Oliveira el al., 1996; López, Flores y Salles, 2000; INEGI, 2001). 
social, y no se ha dado suficiente atención a los aspectos relacionados con el cuerpo, la sexualidad, lo religioso, lo cultural, lo legal, y en particular con los derechos humanos de las mujeres. Además esta especialista observa que el IPM opera en el campo de la elección femenina abierta (su participación en la vida pública) y no busca capturar los factores subyacentes e invisibles de las relaciones de poder, es decir, aquellos que se consideran naturales y obvios. Esta crítica puede parecer injustificada si se tiene en cuenta que resulta poco menos que imposible contar con información comparable a nivel internacional sobre los diferentes aspectos mencionados. ${ }^{7}$ No obstante dicha crítica tiene la virtud de recordarnos las dimensiones involucradas en el proceso de empoderamiento, así como la necesidad, posiblemente insoslayable, de ir más allá de la información disponible y diseñar indicadores particulares sobre dicho proceso.

Una investigación que se propuso ubicar las manifestaciones específicas del proceso de empoderamiento de las mujeres y recibió mucha atención en este campo de estudio fue la llevada a cabo por $\mathrm{M}$. Schuler y S. Hashemi a principios de los noventa en Bangladesh (véase Schuler y Hashemi, 1996). En su estudio sobre los programas de crédito y su impacto sobre las mujeres en este país, estas especialistas instaron a informantes clave entre las participantes en los programas a identificar los cambios indicativos del empoderamiento. De ese inventario surgió un conjunto de seis categorías centrales: 1) sentido de seguridad y visión de un futuro; 2) capacidad para ganarse la vida; 3) capacidad para actuar eficazmente en la esfera pública; 4) mayor poder para tomar decisiones en el hogar; 5) participación en grupos no familiares y uso de grupos de solidaridad como recursos de información y apoyo; 6) movilidad y visibilidad en la comunidad (véase también Schuler, 1997). Si bien aquí se trata sin duda de aspectos más cercanos a las transformaciones que nos interesan, hay que tener en cuenta que se privilegian manifestaciones concretas en las prácticas individuales, y que éstas bien podrían asimilarse también a la acepción que consideramos más apropiada sobre la autonomía femenina.

Un intento más reciente por identificar qué indicadores de empoderamiento estarían en diferentes planos de la realidad es el de Sen y

7 En ausencia de información más específica, es usual que la discusión sobre indicadores de empoderamiento se relacione de manera más directa con la participación femenina en la vida política o pública en general (véase al respecto López y Salles, 1996). 
Batliwala (2000). Estos proponen los siguientes: 1) acciones que las mujeres llevan a cabo -de manera colectiva o individual- para afirmar o imponer sus derechos (diferentes a las prácticas de salud o reproducción que generalmente se miden por medio de encuestas; se trataría más bien de los desafios concretos a los sistemas de poder de género); 2) seguimientos de los sistemas de salud para evaluar en qué medida responden a las preocupaciones y necesidades de las mujeres; 3) cambios en la incidencia de la violencia de género y el abuso; 4) mejoramiento de los indicadores de salud en las áreas clave de la salud sexual y reproductiva. ${ }^{8}$

Estas propuestas pueden considerarse complementarias y corresponderá a cada investigador(a) definir la selección concreta de posibles indicadores que utilizará dependiendo de sus propósitos, de las fuentes de información a su disposición, y de su contexto social y cultural. Se trata más bien de ejemplos ilustrativos de lo que es posible hacer, así como de los alcances y limitaciones de cada elección particular. Todos ellos sugieren también que es posible realizar investigación social sobre el empoderamiento partiendo de los resultados o manifestaciones de dicho proceso en diferentes esferas de la realidad. Esta posición siempre nos ha parecido un punto de partida muy útil para la investigación concreta en torno a las transformaciones complejas y dinámicas. ${ }^{9}$

${ }^{8}$ Dichas autoras complementan estas propuestas en torno a la medición de los resultados del empoderamiento con otra serie de indicadores para evaluar los derechos de las mujeres en un momento determinado. Hacen hincapié en que los derechos sexuales y reproductivos son centrales en el empoderamiento femenino. Los indicadores sobre tales derechos fueron adaptados de la serie elaborada por Jacobson (1991) y se refieren a: la medida en la que las mujeres pueden determinar cuándo y con quién tienen relaciones sexuales; si pueden tener relaciones sexuales sin miedo de infección o embarazos no deseados; si pueden escoger cuándo y cómo regular su fecundidad; si se pueden enfrentar a los embarazos y a los partos de manera segura; si pueden obtener un aborto seguro cuando así lo deseen; si pueden obtener otros servicios de salud sexual y reproductiva cuando los requieren, y si pueden obtener fácilmente información sobre prevención y tratamiento de enfermedades reproductivas con respeto a la confidencialidad (Sen y Batliwala, 2000: 29).

${ }^{9}$ Tiempo atrás Vilmar Faría (1976) planteaba que cuando el nivel de abstracción teórica es muy elevado, muchas veces se tiene que partir de lo que éste implica en torno a algunas manifestaciones concretas, e iniciar alí la investigación empírica. 


\section{Indicadores de empoderamiento y autonomía con base en la información de las encuestas}

A medida que el concepto de empoderamiento gana terreno en la sociodemografia se amplía también el uso de encuestas para intentar acercarse al conocimiento de esta transformación. Es conocida la importante tradición de las encuestas dentro de nuestra disciplina; a lo largo de las últimas décadas han sido llevados a cabo notables y extensos esfuerzos de encuestas comparativas a nivel internacional para documentar los niveles y tendencias de la fecundidad. En la búsqueda de factores asociados a las transformaciones en la fecundidad y la mortalidad, la condición femenina siempre ha sido considerada un eje analítico central, pero la postura más frecuente ha sido referirse al estatus o posición de las mujeres (véase Mason, 1995; Sen y Batliwala, 2000). Es sabido que el estatus hace referencia a diferentes rangos dentro de una jerarquía, y que la información que tradicionalmente se recolecta en los censos y las encuestas permite acercarse satisfactoriamente a este concepto. El estatus de las mujeres en las encuestas demográficas generalmente se mide mediante variables como la educación, la condición de actividad, la ocupación desempeñada, el ingreso, la edad, la diferencia de edad entre la mujer y su cónyuge, y la edad a la unión. ${ }^{10}$

Muchos de estos aspectos se asocian de manera estrecha con las transformaciones demográficas, y la mayor escolaridad femenina es tal vez el mejor predictor existente de una menor mortalidad infantil y de una menor fecundidad. Sin embargo, no siempre la escolaridad y la participación laboral femenina se asocian con el logro de mayor equidad entre hombres y mujeres. Por ejemplo, en algunos países asiáticos no necesariamente la mayor escolaridad se asocia con la mayor independencia en la toma de decisiones en el hogar, tal y como sucede en el caso de América Latina. Esto se debe a que en el mundo islámico las mujeres con mayor escolaridad sufren una reclusión en el hogar más severa, y por lo tanto tienen menor posibilidad de decidir aspectos centrales en sus vidas. De la misma manera, el trabajo extradoméstico femenino en muchas sociedades no necesariamente lleva a que las mujeres sean más independientes de los varones, pues a veces

${ }^{10}$ En la actualidad se discute mucho si este conjun to de variables permite realizar una medición indirecta o directa de la posición de la mujer. Como se verá a continuación, se busca cada vez más acercarse a una medición directa. 
no controlan el dinero que ganan o lo entregan de manera automática a sus esposos o a sus suegras (véase la exposición al respecto en Mason, 1995). De esta suerte, es cada vez más frecuente que aspectos como la escolaridad y el empleo sean considerados referentes indirectos-muchas veces denominados "proxies" en la literatura científica en inglés- del posible empoderamiento y autonomía de las mujeres, y que se dedique tiempo y esfuerzo al diseño de indicadores directos.

¿Qué aspectos se privilegian, o con qué tipo de información se cuenta más frecuentemente para estos indicadores directos? ¿Se justifica en este caso que muchos autores se refieran a ellos como indicadores directos de autonomía -o incluso de eslatus o posición-y no tanto de empoderamiento? En el Apéndice se listan varias investigaciones recientes que recurren al diseño de indicadores directos basados en encuestas. Mediante el análisis de esta información puede corroborarse que una buena cantidad de especialistas escoge el término de indicadores directos de autonomía, o también de estatus o posición; sin embargo no falta quien también sostenga que se trata de indicadores de empoderamiento o del acceso a las fuentes de poder. A pesar de esta diversidad, hay mucha coincidencia en las dimensiones que se pretende medir, y éstas apuntan en mayor medida a las manifestaciones concretas de independencia, al control de la propia vida y (o) a la actuación según intereses propios. Por lo anterior, coincidimos con aquellos que seleccionan como más apropiado el término de indicadores directos de autonomía para referirse a los aspectos que se mencionan a continuación. ${ }^{11}$ Éstos son:

- Participación de la mujer en la toma de decisiones en el hogar. Hay que recordar que esta dimensión tiene mucha tradición en los estudios sociodemográficos y ha sido frecuentemente postulada como clave en el control de la conducta reproductiva y el logro de una menor fecundidad. ${ }^{12}$ En este caso, lo que más conviene tener en cuenta para evaluar la autonomia es la participación femenina en las decisiones referentes a la educación, la salud y el matrimonio de los hijos e hijas, el

"AJgunos indicadores, como el acceso y control de los recursos económicos (véase más adelante), bien pueden aproximarse indistintamente a varios de los conceptos que hemos manejado. Este caso muestra los limites de las separaciones conceptuales y analíticas que venimos mencionando.

12 Kritz, Makinwa Adebusoye y Gurak (2000) identifican como referencias pioneras en la discusión de los problemas metodológicos relacionados con el concepto de toma decisiones en el hogar a los siguientes trabajos: Bagozzi y Van Loo (1980), Beckman (1982), Hollerbach (1980) y Stark (1984). 
uso de anticonceptivos y la planificación familiar, así como la influencia de la mujer en lo concerniente a las compras y los gastos del hogar.

- Libertad de movimiento. Otro aspecto que surge recurrentemente es la libertad de movimiento fuera del hogar y de la localidad de residencia, y por consiguiente la necesidad o no de pedir permiso para efectuar estos desplazamientos. La frecuencia con que se menciona esta dimensión podría estar relacionada con el hecho de que gran parte de los autores que analizamos ha llevado a cabo sus investigaciones en la región asiática, donde la reclusión femenina es un aspecto crucial de la diferenciación de género. Sin embargo, trabajos como los de Casique (2001a y 2001b) y García y Oliveira (2000) para el caso de México han mostrado la relevancia de esta dimensión, aun en el caso de las áreas urbanas más importantes de nuestro país. Asimismo se ha observado que el porcentaje de mujeres que necesitan pedir permiso en México varía muy poco al ser reportado por hombres o por mujeres (García y Oliveira, 2000).

- Acceso y control de recursos económicos. Aun cuando en este aspecto no existe tanta coincidencia como en los anteriores en lo relativo a las preguntas o indicadores más específicos -los cuales necesariamente varían según el contexto en cuestión-, resulta claro que el acceso y control del dinero u otros objetos de valor son también considerados clave en la lucha por superar la subordinación femenina. Por lo general se intenta ir más allá del desempeño de un trabajo extradoméstico, y más bien se busca detectar en qué medida la mujer efectivamente aporta, controla o puede responder aunque sea parcialmente por su manutención económica y la de su familia.

- Estar libre de violencia doméstica. Este aspecto se menciona menos que los demás, pero nos parece que los intentos en esta dirección merecen ser destacados porque la violencia doméstica contra la mujer es una de las dimensiones menos evidentes -pero no por ello menos amenazadoras- de la desigualdad entre hombres y mujeres cuando se aspira a ejercer control sobre la propia vida. Cabe destacar que se considera relevante detectar tanto el estar libre de amenazas y miedo, como la ausencia misma de la violencia física por parte del esposo o compañero.

- Actitudes a favor de la equidad de género. La dimensión subjetiva, esto es, las percepciones y actitudes sobre la desigualdad entre hombres y mujeres y sobre el derecho que ellas tienen a realizar diversos tipos de actividades también son considerados relevantes entre los indicadores directos de autonomia. Se tienen en cuenta las actitudes respecto al desempeño de tareas extradomésticas, la salud propia y de los hijos, el uso de anticonceptivos, la planificación familiar en gene- 
ral, y las decisiones respecto al presupuesto del hogar, entre otros aspectos. La identificación de este tipo de indicadores responde a la inquietud por acercarse a la dimensión interior, personal, cognitiva, de concientización sobre los problemas de género.

- Elección del cónyuge, composición de la pareja y del hogar. Dentro de este rubro consideramos una serie de indicadores referidos a poder o no elegir al cónyuge, a la diferencia de edad entre los esposos, a la existencia de algún tipo de parentesco entre los miembros de la pareja, y a la convivencia con la familia política. Desde nuestra perspectiva, algunos de estos indicadores -como el poder o no elegir al cónyuge- estarían mejor ubicados que otros en esta lista de indicadores más específicos. No obstante, todos parecen relacionarse de manera muy estrecha con la automomía de la mujer, de ahí que los estudiosos a veces los consideren de manera indistinta como indicadores directos.

Este conjunto de elementos ha permitido acumular conocimientos sobre los niveles de autonomia femenina en diferentes regiones del mundo, lo cual, en sí mismo, debería en principio considerarse como un objeto de estudio legítimo. Sin embargo hay que reconocer que uno de los propósitos centrales de muchos especialistas en estudios de población es investigar la posible influencia del empoderamiento y (o) autonomia de las mujeres sobre las transformaciones demográficas, más allá de lo que hoy se"conoce mediante indicadores como la escolaridad o la participación laboral femenina. La mayor cantidad de investigaciones existentes se centra en los patrones reproductivos (número de hijos, preferencias de fecundidad, deseo de no tener más hijos, uso y tipo de anticonceptivos) y en la salud y el bienestar infantil (sobrevivencia de los hijos, atención médica pre y postnatal, asistencia escolar) (véase el Apéndice). La relación inversa, esto es, la medida en que los cambios en la conducta reproductiva y en la salud de los hijos afectan o pueden ser considerados como indicadores de empoderamiento y (o) autonomía de las mujeres es abordada de manera menos frecuente en la sociodemografia, pero sabemos que está muy presente en varios esfuerzos de conceptuación en este campo (véase la exposición que presentamos arriba). La existencia de estas relaciones recíprocas constituye un reto adicional para la investigación sociodemográfica, especialmente si se utilizan herramientas estadísticas como la regresión lineal o logística. ${ }^{13}$

13 Véase Balk, 1994 y Casique, 200la para el análisis estadístico; Mason, 1995 y Cosío-Zavala, 1997 para la discusión conceptual y (o) la presentación de algunos estudios empíricos en esta doble dirección. 
Al investigar la influencia de la autonomía de la mujer sobre los patrones reproductivos se han encontrado evidencias de que, en efecto, a igualdad de condiciones de escolaridad y de diversas variables demográficas y socioeconómicas, una mayor autonomía individual femenina está relacionada con los deseos y prácticas en torno a un número reducido de hijos. Sin embargo los resultados no son siempre uniformes, y esto suele atribuirse en parte a la multidimensionalidad de las transformaciones objeto de estudio (tanto por el lado de la autonomía como por el de los cambios poblacionales). También influye -según varios especialistas- el hecho de que las variables individuales de autonomía pueden operar de manera distinta en las comunidades o países caracterizados por diferentes sistemas de género. ${ }^{14}$ En el caso de la salud y el bienestar infantil los resultados suelen ser más contundentes al demostrar la importancia de la autonomia de la mujer en esta relevante área, pero aquí también están presentes los aspectos de multidimensionalidad antes mencionados.

\section{Síntesis y consideraciones finales}

En este artículo hemos analizado los conceptos de empoderamientoy autonomia de las mujeres en un esfuerzo por contribuir a precisar su significado e importancia, así como de avanzar en su estudio y medición. En primer lugar abordamos los antecedentes del término empowerment (empoderamiento, en nuestro caso), y vimos que surge estrechamente ligado a la acción y a los movimientos sociales que desafian las fuentes de poder y buscan cambiar la distribución del mismo. Su uso fue plenamente incorporado en la investigación y en las políticas de población a raíz de la Conferencia Internacional de Población y Desarrollo de 1994, que fue la primera conferencia internacional de población donde las activistas a favor de la salud de las mujeres tuvieron un papel preponderante.

En términos conceptuales indicamos que no todos los autores/as están de acuerdo respecto a cuáles son los aspectos que deben ser tomados en cuenta en la definición de empoderamiento. Coincidimos con la

${ }^{14}$ Estas apreciaciones están basadas en la revisión de los estudios listados en el Apéndice. La combinación de las perspectivas individual y contextual está presente en: Balk, 1994; Durrant y Sathar, 2000; Jejeebhoy, 2000 y 2001; Jejeebhoy y Sathar, 2001; Kritz, Makinwa-Adebusoye y Gurak, 2000; Mason y Smith, 1999; Morgan y Niraula, 1995; Niraula y Morgan, 2000; Morgan, Stash, Smith y Mason, 2002; Sathar, Callum y Jejeebhoy, 200I; Schuler y Hashemi, 1994 y Uchudi, 2000. 
posición de que el empoderamiento no es sólo la potenciación, sino la lucha por el acceso a las fuentes de poder y el control de muy diferentes tipos de recursos (humanos, físicos, ideológicos). Dado que se trata de una transformación muy compleja, documentamos diversos esfuerzos por dar cuenta de sus facetas y componentes o dimensiones. Asimismo analizamos algunas perspectivas conceptuales que clarifican la naturaleza y el tipo de poder que estaría detrás del empoderamiento de las mujeres.

Respecto a las diferencias entre el empoderamiento y la autonomía, analizamos los planteamientos de diversas autoras. Por una parte nos pareció discutible que la autonomía pudiese ser definida sólo como la faceta psicológica del empoderamiento, o que ambos aspectos hiciesen alusión a esferas diferenciadas de la vida familiar y las capacidades individuales. Asimismo no nos pareció enteramente convincente que el empoderamiento y la autonomía pudiesen ser vistos como distintos porque operaban en diferentes planos de la realidad, o porque el primero fuese un concepto dinámico y el otro estático. Sin embargo, rescatamos de varias de estas posiciones la búsqueda implícita o explícita de eslabonamiento o convergencia entre las transformaciones objeto de atención. Lo anterior nos llevó a señalar que la autonomía (independencia y actuación según intereses propios) es una de las posibles manifestaciones del empoderamiento (desafio y eventual acceso a las fuentes de poder y al control de diferentes tipos de recursos), y que puede tener lugar en los niveles social o individual a lo largo del tiempo.

En el terreno metodológico hicimos hincapié en los esfuerzos desempeñados por distintos especialistas y organismos internacionales para diseñar indicadores en este campo de interés, ya sea con base en la información existente o en estudios especialmente diseñados. Recibió atención particular el papel de los indicadores directos producto de la información de encuestas, a los que en principio se les denomina de estatus, empoderamiento o autonomía. No obstante esta diversidad, las áreas específicas que se tienen en cuenta son muy semejantes, y consideramos que estos indicadores se aproximarían más en su conjunto al concepto de autonomía. Ellos son: participación femenina en la toma de decisiones en el hogar, libertad de movimientos, acceso y control de los recursos económicos, estar libre de violencia doméstica, actitudes en favor de la equidad de género, y diversos aspectos relacionados con la elección del cónyuge y la composición de la pareja y el hogar.

Este conjunto de reflexiones demuestra de diversas maneras cómo el estudio del empoderamiento y de la autonomía de las mujeres 
nos lleva a dar un paso adelante en el desarrollo de la perspectiva de género en las ciencias sociales en general, y en los estudios de población en particular. En un primer momento la perspectiva de género pretendió mostrar las desigualdades entre hombres y mujeres y la contribución femenina a la producción y reproducción de la sociedad. En etapas posteriores las acciones emprendidas por el movimiento de mujeres han hecho ver la importancia de abordar aspectos concretos del desafio al status quo, de la búsqueda por cambiar la distribución del poder y el control de los recursos y la ideología, así como las manifestaciones de estos procesos en cuanto a independencia y actuación personal. A partir de aquí los avances en el terreno metodológico han sido significativos, especialmente en lo concerniente al diseño de indicadores específicos o directos basados en encuestas, de los cuales apuntamos sus posibilidades y limitaciones.

Los procesos de empoderamiento y (o) autonomia de las mujeres constituyen sin duda alguna objetos de estudio legítimos en sí mismos, pero también suelen considerarse relevantes en la explicación de los fenómenos demográficos. El reto metodológico y técnico para la investigación en este campo ha sido demostrar que efectivamente esta influencia existe, más allá de lo que ya se sabía mediante el análisis tradicional de la escolaridad y de la participación femenina en el mercado de trabajo. El lector encontrará en este artículo varios ejemplos de este tipo de investigación más reciente, que esperamos coadyuven a orientar su propia búsqueda y contribución a la perspectiva de género en los estudios de población. 


\section{Apéndice}

Investigaciones recientes que se basan en indicadores directos de estatus, autonomía o empoderamiento con información de encuestas ${ }^{15}$

Balk, Deborah (1994), "Individual and Community Aspects of Women's Status and Fertility in Rural Bangladesh", Population Studies, núm. 48, pp. 21-45.

Variables de estatus de la mujer a nivel individual y de la comunidad

A nivel individual:

- Movilidad (frecuencia de viajes fuera de la comunidad, uso de la burka, posibilidad de salir sola o acompañada por niños, parientes mujeres, parientes hombres o esposos).

- Actividades que le son permitidas a la mujer en el hogar (adoptar la planificación familiar, recrearse, ganar dinero, llevar a un niño enfermo a un hospital fuera de la comunidad).

- Autoridad en la toma de decisiones en el hogar (participación en la toma de decisiones respecto a comprar medicinas para un hijo enfermo, ver a un médico cuando está enferma, cuánto tiempo debe un niño asistir a la escuela, a qué edad y con quién se casa una hija, gastar el dinero que ella gana, gastar el dinero que gana el esposo; actitudes sobre adoptar métodos de planificación familiar aun cuando el esposo se oponga).

- Actitudes respecto a: viajar sola, decidir sobre tratamientos médicos para ella o para los hijos enfermos, trabajar fuera del hogar para sobrevivir.

A nivel de la comunidad: Indicadores que resumen las cuatro dimensiones anteriores a nivel de las comunidades estudiadas.

15 La gran mayoria de estos indicadores están diseñados con información de la mujer o de la pareja, pero señalamos cuando dicha información se agrega por diferentes medios para conformar contextos a nivel comunitario. 
Casique, Irene (2001a), Power, Autonomy and Division of Labor in Mexican Dual-Earner Families, Lanham, Nueva York, Oxford, University Press of America (véase también Casique, 200lb).

Indicadores del poder y la autonomía de la esposa

Indicadores de poder: Proceso de toma de decisiones (participación en la toma de decisiones respecto a cuántos hijos tener, cómo criarlos, qué gastos diarios llevar a cabo, qué parientes y amigas visitar y cuándo tener relaciones sexuales).

Indicadores de autonomia: Necesidad de contar con el permiso del esposo para: salir fuera de la casa, salir fuera de la casa con niños, decidir sobre los gastos diarios, visitar amigas, visitar parientes, trabajar, estudiar, usar anticonceptivos y participar en las actividades de la comunidad.

Durrant, Valerie L., y Zeba Sathar (2000), "Greater Investments in Children through Women's Empowerment: A Key to Demographic Change in Pakistan?", Nueva York, Policy Research Division Working Papers, The Population Council.

Indicadores del estatus de la mujer a nivel individual y de la comunidad

A nivel individual:

- Purdah fuera de la casa.

- Movilidad (posibilidad de salir sola y sin permiso al mercado, al centro de salud y a la casa de una amiga o pariente).

- Miedo al esposo (tener miedo de estar frecuentemente en desacuerdo con el esposo).

- Violencia doméstica (haber sido golpeada por el esposo).

- Acceso a recursos financieros (tener dinero a mano para gastar; poseer tierra, joyas u otros valores de los que se pueda disponer cuando se desee).

- Toma de decisiones respecto a los niños (participación en decisiones sobre el castigo, qué hacer cuando se enferman, cuánta escolaridad deben recibir y de qué tipo).

- Esposo mayor (si el esposo es cinco o más años mayor que la mujer). 
A nivel de la comunidad:

- Movilidad (medida que resume la movilidad de las mujeres en cada comunidad, excluyendo a la entrevistada).

- Trabajo fuera del hogar (porcentaje de mujeres en la comunidad que trabajan fuera del hogar, excluyendo a la entrevistada).

- Miedo al esposo (porcentaje de mujeres en la comunidad que tienen miedo de estar frecuentemente en desacuerdo con los esposos, excluyendo a la entrevistada).

- Diferencias de edad entre los esposos (medida que resume para la comunidad la diferencia de edad entre las mujeres y sus esposos).

Govindasamy, Pavalavalli (2000), "Poverty, Women's Status, and the Utilization of Health Services in Egypt", en Brígida García (ed.), Wo men, Poverty, and Demographic Change, Oxford, Oxford University Press, pp. 263-285.

Indicadores de la posición de las mujeres en Egipto

- Participación en la decisión de tener otro hijo.

- Libertad de movimientos.

- El esposo es pariente.

- Opinión sobre si las esposas deberían expresar su punto de vista.

- Opinión sobre si las esposas deberían trabajar (trabajo extradoméstico).

- Opinión sobre la última palabra en torno al presupuesto familiar.

- Opinión sobre la última palabra en torno al uso de la planificación familiar.

Jejeebhoy, Shireen J. y Zeba A. Sathar (2001), "Women's Autonomy in India and Pakistan: The Influence of Religion and Region", Population and Development Review, vol. 27, núm. 4, pp. 687-712 (véase también Jejeebhoy, 2000 y 2001; Sathar, Callum y Jejeebhoy, 2001).

Indicadores de autonomía femenina en India y Pakistán

- Autoridad en las decisiones económicas (participa en las decisiones de compra de alimentos, bienes importantes para el hogar y joyas).

- Autoridad en las decisiones relacionadas con los hijos (partici- 
pa en las decisiones sobre qué hacer cuando están enfermos, la disciplina, la educación y el tipo de escuela al que deben asistir).

- Movilidad (puede ir sin acompañante a lugares como los centros de salud, los centros comunitarios, el hogar de una pariente o amiga, una feria o el próximo poblado).

- Estar libre de amenazas (no le tiene miedo al esposo y no ha sido golpeada).

- Acceso y control de recursos económicos (opina sobre el gasto del hogar, tiene dinero para gastar, es libre para comprar pequeñas joyas, es libre para comprar regalos para parientes, los objetos de valor del hogar le pertenecen y son controlados por ella, planea usar sus ahorros para apoyo futuro, tiene qué decir en cómo se gasta la dote).

Kishor, Sunita (2000a), "Empowerment of Women in Egypt and Links to the Survival and Health of Their Infants", en Harriet Presser y Gita Sen (eds.), Women's Empowerment and Demographic Processes. Moving Beyond Cairo, Oxford, Oxford University Press, pp. 119-156.

Factores para las diferentes dimensiones del empoderamiento en Egipto ${ }^{16}$

- Autonomía financiera.

- Participación en el sector modemo (relacionado con la posesión de bienes, educación, exposición a medios, cuentas de banco y otros indicadores).

- Exposición a un empleo a lo largo de la vida.

- Compartir roles y toma de decisiones.

- Estructura familiar favorable al empoderamiento (relacionado con el no vivir o haber vivido con la familia política, controlar gastos, compartir decisiones).

- Igualdad en el matrimonio.

- Devaluación de las mujeres (relacionado con aspectos como la violencia doméstica, la importancia de la dote y otros indicadores).

${ }^{16}$ Se refiere a 10 factores identificados mediante un análisis factorial, que resume una serie muy amplia de indicadores'sobre tres aspectos del empoderamiento: a) evidencia de empoderamiento; b) fuentes de empoderamiento; c) escenario (selting) del empoderamiento. Cuando el nombre dado a los factores no se explica por sí mismo, hago referencia a las variables con las que están relacionados; para mayor información véase Kishor, 2000a. 
- Emancipación de las mujeres (relacionado con la educación de las hijas, la libertad de movimientos y el trabajo para el autodesarrollo).

- Ventajas maritales (relacionado con la poca diferencia de edad entre los cónyuges, poder elegir el cónyuge, estar casada con un pariente, edad al matrimonio y otros indicadores).

- Matrimonio tradicional (relacionado con la poca diferencia educacional y el estar casada con un pariente).

Kishor, Sunita (2000b), 'Women's Contraceptive Use in Egypt: What Do Direct Measures of Empowerment Tell Us?", trabajo presentado en la Reunión Anual de la Population Association of America (PAA).

Indicadores sobre la evidencia de empoderamiento en Egipto

- Toma de decisiones en el hogar (participación en las decisiones respecto a los alimentos cocinados, uso de anticonceptivos, tener otro hijo, medicina de los hijos, educación de los hijos, presupuesto, visitas a amigas y $(0)$ familiares.

- Libertad de movimientos (posibilidad de ir sola o acompañada por los hijos: sólo fuera de la casa, al mercado local, a los hogares de amigas o familiares, al centro local de salud, a lugares de recreo).

Kritz, Mary M., Paulina Makinwa Adebusoy y Douglas T. Gurak (2000), "The Role of Gender Context in Shaping Reproductive Bahaiour in Nigeria", en Harriet Presser y Gita Sen (eds.), Women's Empowerment and Demographic Processes. Moving Beyond Cairo, Oxford, Oxford University Press, pp. 239-260.

Indicadores de autonomia individual y efectos en el nivel del contexto macro

Autonomía individual:

- Toma de decisiones (respecto a las compras del hogar, el trabajo extradoméstico femenino, cómo se gasta el ingreso del marido, el número de hijos que se tienen, si se compra o se vende la tierra, si se usa la planificación familiar, si se envían los hijos a la escuela, qué tanta educación deben recibir los hijos, cuándo se deben casar los hijos varones, cuándo se deben casar las hijas mujeres, si se lleva un hijo enfermo al doctor, y cómo criar a los hijos). 
- Contribución financiera de la esposa (si la esposa pagó más que el esposo en la alimentación, la vestimenta de los hijos, las colegiaturas y la salud).

Efectos contextuales de equidad de género: Incluye indicadores para distintas áreas geográficas elaborados a partir de variables individuales o de la pareja: diferencia media de edad entre los esposos, porcentajes de esposos y esposas que están de acuerdo con que la esposa tenga la voz final en las decisiones del hogar o con que ambos tomen las decisiones finales, porcentaje de esposas que escogen a sus esposos, porcentaje de esposas que tienen trabajo extradoméstico moderno, porcentaje de esposas que deciden cómo se gasta su ingreso, porcentaje de esposas que han experimentado una muerte infantil, índice medio de movilidad fisica de la esposa, y porcentaje de mujeres en la actividad comercial o agrícola).

Kulczycki, Andrzej y Lucía Juárez (2003), "The Influence of Female Employment and Autonomy on Reproductive Behaviour in Egypt", en Brígida García, Richard Anker y Antonella Pinnelli (eds.), Women in the Labour Market in Changing Economies: Demographic Issues, Oxford, Oxford University Press, pp. 314-330.

Indicadores de autonomía de la mujer en Egipto

- Poder en las decisiones financieras (tiene la decisión final en el presupuesto familiar).

- Poder en las decisiones reproductivas (influye en la decisión de tener un hijo adicional).

Mason, Karen Oppenheim y Herbert L. Smith (1999), "Female Autonomy and Fertility in Five Asian Countries", trabajo presentado en la Reunión Anual de la Population Association of America (PAA).

Indicadores de autonomía de la mujer en cinco países asiáticos

- Poder o autonomía en las decisiones económicas (participación en las decisiones sobre las compras de objetos de valor y el trabajo femenino extradoméstico; sentirse libre de comprar vestidos o pequeñas joyas sin el consentimien to del esposo o de un miembro mayor de la familia). 
- Poder o autonomía en las decisiones reproductivas (participación en las decisiones sobre cuántos hijos tener).

- Libertad de movimientos (necesidad de obtener permiso para ir al mercado local, al centro de salud local, a los campos fuera del poblado de residencia, al centro comunitario del poblado, al hogar de una pariente o amiga, a una feria cercana, a un templo o al próximo poblado).

- Temer al esposo (sentir miedo de estar en desacuerdo con el esposo por temor a su enojo).

- El esposo golpea a la esposa (haber sido golpeada por el esposo alguna vez en la vida).

Morgan, S. Philip, Sharon Stash, Herbert L. Smith y Karen Oppenheim Mason (2002), "Muslim and Non-Muslim Differences in Female Autonomy and Fertility: Evidence from Four Asian Countries", Population and Development Review, vol. 28, núm. 3, pp. 515-537.

Indicadores de autonomía en cuatro países asiáticos

— Índice de libertad de movimiento (no ser libre para ir a todos los lugares que uno escoja).

- Índice de poder económico (compras de ropa y joyas).

- Índice de no coerción (temor de estar en desacuerdo con el esposo).

Niraula, Bhanu B. y S. Philip Morgan (2000), "Gender Inequality in Two Nepali Settings", en Brígida García, Women, Poverty, and Demographic Change. Oxford, Oxford University Press, pp. 42-72 (véase también Morgan y Niraula, 1995).

Indicadores de autonomía de la mujer en Nepal

- Libertad de movimiento (si es aceptable o no ir sola al centro local de salud, al mercado local, a visitar parientes, a los campos situados fuera del poblado de residencia, al centro comunitario, a la feria o al templo, al próximo poblado y al cine).

- Influencia en la toma de decisiones en el hogar (participación en las decisiones sobre tener otro hijo, buscar tratamiento para un 
hijo varón, buscar tratamiento para una hija, castigar a un hijo, castigar a una hija, cuánta escolaridad es conveniente para un hijo, cuánta escolaridad para una hija, qué alimentos comprar, si debe trabajar fuera del hogar y si debe comprar o vender objetos de valor).

Schuler, Sidney Ruth y Syed M. Hashemi (1994), "Credit Programs, Women's Empowerment and Contraceptive Use in Rural Bangladesh", Studies in Family Planning, vol. 25, núm. 2, pp. 65-76.

Indicadores del nivel de empoderamiento de las mujeres

- Movilidad.

- Seguridad económica.

- Pequeñas y grandes compras.

- Decisiones mayores.

- Dominación y violencia (estatus y poder de decisión económica en el hogar).

- Esferas política y legal (habilidad para interactuar efectivamente en la esfera pública).

- Protestas y campañas (participación en grupos no familiares).

\section{Bibliografía}

Ariza, Marina y Orlandina de Oliveira (1996), "Propuesta de un marco analíti-. co general de las inequidades de género y clase", en Orlandina de Oliveira et al., La condición femenina: una propuesta de indicadores, México, Sociedad Mexicana de Demografia (Somede)/Consejo Nacional de Población (Conapo) (inédito).

Bagozzi, R. P. y M. F. Van Loo (1980), "Decision-Making and Fertility: A Theory of Exchange in the Family", en T. K. Burch (ed.), Demographic Behavior, Boulder, Colorado, Westview Press, pp. 91-124.

Beckman, L. J. (1982), "Communication, Power, and the Influence of Social Networks in Couple Decisions on Fertility", en R. A. Bulatao y R. D. Lee (eds.), Determinants of Fertility in Developing Countries, Nueva York, Academic Press, pp. 415-443.

Balk, Deborah (1994), "Individual and Community Aspects of Women's Status and Fertility in Rural Bangladesh", Population Studies, núm. 48, pp. 21-45.

Batliwala, Srilatha (1994), "The Meaning of Women's Empowerment: New Concepts from Action", en Gita Sen, Adrienne Germain y Lincoln C. Chen (coords.), Population Policies Reconsidered. Health, Empowerment and Rights, Boston, 
Harvard Center for Population and Development Studies/International Women's Health Coalition, pp.127-138 [traducción al español: Batliwala, Srilatha (1997), "El significado del empoderamiento de las mujeres: nuevos conceptos desde la acción", en Magdalena León (comp.) (1997a), Poder y empoderamiento de las mujeres, Bogotá, Tercer Mundo Editores/Facultad de Ciencias Humanas de la Universidad Nacional de Colombia, pp. 187-211].

Bookman, A. y S. Morgen (1988), Women and the Politics of Empowerment, Filadelfia, Temple University Press.

Casique, Irene (2001a), Power, Autonomy and Division of Labor in Mexican DualEamer Families, Lanham, University Press of America.

(2001b), "What Difference Does It Make? Women's Autonomy and Power and Use of Contraception in Mexico", trabajo presentado en la XXIV Conferencia General de Población de la International Union for the Scientific Study of Population (IUSSP), Salvador, Bahía, Brasil (mimeo.).

Cosío-Zavala, María Eugenia (ed.) (1997), Women and Families: Evolution of the Status of Women as a Factor and Consequence of Changes in Family Dynamics, París, Committee for International Cooperation in National Research in Demography (CICRED)/United Nations Population Fund (UNFPA)/Permanent Mexican Delegation at UNESCo (United Nations Educational, Scientific and Cultural Organization).

Durrant, Valerie L. y Zeba Sathar (2000), "Greater Investments in Children through Women's Empowerment: A Key to Demographic Change in Pakistan?", Nueva York, The Population Council (Policy Research Division Working Papers).

Faría, Vilmar (1976), Occupational Marginality, Employment and Poverty in Urban Brazil, tesis de doctorado, Cambridge, Mass., Universidad de Harvard.

Freire, Paulo (1973), The Pedagogy of the Opressed, Nueva York, Seabury Press [versión en español: Freire, Paulo (1986), La pedagogía de los oprimidos, México, Siglo XxI].

Foucault, Michel (1980), Power/Knowledge. Selected Interviews and Other Writings 1972-1977, Nueva York, Pantheon. (1972), The Archaeology of Knowledge, Nueva York, Pantheon.

Govindasamy, Pavalavalli (2000), "Poverty, Women's Status, and the Utilization of Health", en Brígida García (coord.), Women, Poverly, and Demographic Change, Oxford, Oxford University Press, pp. 263-285.

García, Brígida y Orlandina de Oliveira (2000), "Dinámica familiar en la Ciudad de México y Monterrey”, Informe final de investigación, México, El Colegio de México (inédito).

Gramsci, A. (1971), Selections from the Prison Notebooks, Nueva York, International Publishers.

Hollerbach, P. E. (1980), "Power in Families, Communication and Fertility Decision-Making", Population and Environment, vol. 3, núm. 2, pp. 146-173.

INEGI (Instituto Nacional de Estadística, Geografia e Informática) (2001), Sistema de indicadores para el seguimiento de la situación de la mujer en México, México, INEGI/UNIFEM/PNUD/UNCEF/FNUAP/OMS/OPS. 
Jejeebhoy, Shireen (2001), "Women's Autonomy and Reproductive Behaviour in lndia", en J. Phillips y Z. Sathar (eds.), Fertility Transition in South Asia, Oxford, Oxford University Press.

(2000), "Women's Autonomy in Rural India: lts Dimensions, Determinants, and the Influence of Context", en Harriet Presser y Gita Sen (eds.), Women's Empowerment and Demographic Processes. Moving Beyond Cairo, Oxford, Oxford University Press, pp. 204-238.

— y Zeba A. Sathar (2001), "Women's Autonomy in India and Pakistan; The Influence of Religion and Region", Population and Development Review, vol. 27, núm. 4, pp. 687-712.

Kabeer, Naila (1994), "Empowerment from Below: Learning from the Grassroots", en Naila Kabeer, Reversed Realities: Gender Hierarchies in Development Thought, Londres, Verso (cap. 9) [traducción al español: Kabeer, Naila (1998), "El empoderamiento desde abajo: lecciones de las bases populares", en Naila Kabeer, Realidades trastocadas. Las jerarquias de género en el pensamiento del desarrollo, México, Paidós/Programa Universitario de Estudios de Género (PUEG)/Instituto de Investigaciones Económicas, UNAM (cap. 9)].

Kishor, Sunita (2000a), "Empowerment of Women in Egypt and Links to the Survival and Health of Their Infants", en Harriet Presser y Gita Sen (eds.), Women's Empowerment and Demographic Processes. Moving Beyond Cairo. Oxford, Oxford University Press, pp. 119-156.

(2000b), "Women's Contraceptive Use in Egypt: What Do Direct Measures of Empowerment Tell Us?", trabajo presentado en la Reunión Anual de la Population Association of America (PAA) (mimeo.).

Kritz, Mary M., Paulina Makinwa Adebusoy y Douglas T. Gurak (2000), "The Role of Gender Context in Shaping Reproductive Bahaiour in Nigeria", en Harriet Presser y Gita Sen (eds.), Women's Empowerment and Demographic Processes. Moving Beyond Cairo, Oxford, Oxford University Press, pp. 239-260.

Kulczycki, Andrzej y Lucía Juárez (2003), "The Influence of Female Employment" and Autonomy on Reproductive Behaviour in Egypt", en Brigida García, Richard Anker y Antonella Pinnelli (eds.), Women in the Labour Market in Changing Economies: Demographic Issues. Oxford, Oxford University Press, pp. 314-330.

León, Magdalena (comp.) (1997a), Poder y empoderamiento de las mujeres, Bogotá, Tercer Mundo/Facultad de Ciencias Humanas de la Universidad Nacional de Colombia.

- (1997b), "El empoderamiento en la teoría y práctica del feminismo", en Magdalena León (comp.), Poder y empoderamiento de las mujeres, Bogotá, Tercer Mundo/Facultad de Ciencias Humanas de la Universidad Nacional de Colombia, pp. 1-26.

López, Ma. de la Paz (coord.), Julia Flores y Vania Salles (2000), "El observatorio de la condición de la mujér en México. La construcción cultural de la diferencia entre los sexos. Análisis con base en las percepciones sociales", Primer informe. Resultados de investigación para discusión, Fundación MacArthur/UNIFEM/Colmex-CES/GIMTRAP (mimeo.). 
López, María de la Paz y Vania Salles (1996), "Participación política, poder y empoderamiento: una discusión desde la óptica de género", en Orlandina de Oliveira et al. (coords.), La condición femenina: una propuesta de indicadores, México, Sociedad Mexicana de Demografia (Somede)/Consejo Nacional de Población (Conapo) (inédito).

Lukes, S. (1974), Power: A Radical View, Londres, MacMillan.

(ed.) (1986), Power, Readings in Social and Political Theory, Oxford, Basil Blackwell.

Mason, Karen Oppenheim (1995), "Gender and Demographic Change: What Do We Know?", Lieja, International Union for the Scientific Study of Population (IUSSP).

_ y Herbert L. Smith (1999), "Female Autonomy and Fertility in Five Asian Countries", trabajo presentado en la Reunión Anual de la Population Association of America (PAA) (mimeo.).

Molyneux, Maxine (1985), “Mobilization without Emancipation? Women's Interests, State and Revolution in Nicaragua", Feminist Studies, vol. 11, pp. 227-254 [traducción al español: Molyneux, Maxine (1994), "¿Movilización sin emancipación? Los intereses de la mujer. Estado y revolución en Nicaragua", Desarrollo y Sociedad, núm. 13, Bogotá, CEDE].

Morgan, S. Philip, Sharon Stash, Herbert L. Smith y Karen Oppenheim Mason (2002), "Muslim and Non-Muslim Differences in Female Autonomy and Fertility: Evidence from Four Asian Countries", Population and Development Review, vol. 28, núm. 3, pp. 515-537.

_ y Bhanu B. Niraula (1995), "Gender Inequality and Fertility in Two Nepali Villages", Population and Development Review, vol. 21, pp. 541-561.

Moser, Caroline (1989), "Gender Planning in the Third World", World Development, vol. 17, núm. 11 [traducción al español: Moser, Caroline (1991), "Planeación de género en el Tercer Mundo: enfrentando las necesidades prácticas y estratégicas de género", en Guzmán et al. (eds.), Una nueva mirada: género en el desarrollo, Lima, Flora Tristán/Entre Mujeres].

Niraula, Bhanu B. y S. Philip Morgan (2000), "Gender Inequality in Two Nepali Settings", en Brígida García (coord.), Women, Poverty, and Demographic Change, Oxford, Oxford University Press, pp. 42-72.

Presser, Harriet (1997), "Demography, Feminism and the Science-Policy Nexus", Population and Development Review, núm. 2, pp. 295-332.

y Gita Sen (eds.) (2000a), Women's Empowerment and Demographic Processes. Moving Beyond Cairo, Oxford, Oxford University Press.

- y Gita Sen (2000b), "Women's Empowerment and Demographic Processes: Laying the Groundwork", en Harriet Presser y Gita Sen (eds.), Women's Empowerment and Demographic Processes. Moving Beyond Cairo, Oxford, Oxford University Press, pp. 3-11.

Riger, Stephanie (1997), "¿Qué está mal con el empoderamiento?", en Magdalena León (comp.), Poder y empoderamiento de las mujeres”, Bogotá, Tercer Mundo/Facut tad de Ciencias Humanas de la Universidad Nacional de Colombia, pp. 55-74. 
Rowlands, Jo (1997), "Empoderamiento y mujeres rurales en Honduras: un modelo para el desarrollo", en Magdalena León (comp.), Poder y empoderamiento de las mujeres, Bogotá, Tercer Mundo/Facultad de Ciencias Humanas de la Universidad Nacional de Colombia, pp. 213-245.

Sathar, Z., C. Callum y S. Jejeebhoy (2001), "Gender, Region, Religion and Reproductive Behavour in India and Pakistan", trabajo presentado en la XxIV Conferencia General de la International Union for the Scientific Study of Population (IUSSP), Salvador, Bahía, Brasil.

Schuler, Margaret (1997), "Los derechos de las mujeres son derechos humanos: la agenda internacional del empoderamiento", en Magdalena León (comp.), Poder y empoderamiento de las mujeres, Bogotá, Tercer Mundo/Facultad de Ciencias Humanas de la Universidad Nacional de Colombia, pp. 29-54.

Schuler, Sidney Ruth y Syed M. Hashemi (1996), "Rural Credit Programs and Women's Empowerment in Bangladesh", World Development, vol. 24, núm. 4, pp. 635-653.

y Syed M. Hashemi (1994), "Credit Programs, Women's Empowerment and Contraceptive Use in Rural Bangladesh", Studies in Family Planning, vol. 25, núm. 2, pp. 65-76.

Sen, Gita (1998), "El empoderamiento como un enfoque a la pobreza", en Irma Arriagada y Carmen Torres (eds.), Género y potreza. Nuevas dimensiones. Santiago de Chile, Isis Internacional, pp. 121-139 (Ediciones de las Mujeres, 26). y Srilatha Batliwala (2000), "Empowering Women for Reproductive Rights", en Harriet Presser y Gita Sen (eds.), Women's Empowerment and Demographic Processes. Moving Beyond Cairo, Oxford, Oxford University Press, pp. 15-36.

- y Karen Grown (1987), Development, Crises and Alternative Visions, Nueva York, Monthly Review Press [traducción al español: Sen, Gita y Karen Grown (1988), Desarrollo, crisis y enfoques alternativos: perspectivas para la mujer en el Tercer Mundo, México, El Colegio de México, PIEM].

Stark, O. (1984), "Bargaining, Altruism, and Demographic Phenomena, Population and Development Review, vol. 10, núm. 4, pp. 679-692.

Stromquist, Nelly (1997), "La búsqueda del empoderamiento: en qué puede contribuir el campo de la educación", en Magdalena León (comp.), Poder y empoderamiento de las mujeres, Bogotä, Tercer Mundo/Facultad de Ciencias Humanas de la Universidad Nacional de Colombia, pp. 75-95.

Uchudi, Joseph (2000), "Gender Contex and Fertility Differences in Sub-Saharan, Africa", trabajo presentado en la reunión anual de la Population Association of America (PAA) (mimeo.).

UNICEF (1997), "El marco conceptual de igualdad y empoderamiento de las mujeres", en Magdalena León (comp.), Poder y empoderamiento de las mujeres, Bogotá, Tercer Mundo/Facúltad de Ciencias Humanas de la Universidad Nacional de Colombia, pp. 173-186.

Venier, Martha Elena (1996), “¿Por qué apoderar?”, Boletin Editorial de El Colegio de México, núm. 67, mayo-junio, pp. 17-20. 
Wieringa, Saskia (1997), "Una reflexión sobre el poder y la medición del empoderamiento de género del PNUD", en Magdalena León (comp.), Poder y empoderamiento de las mujeres, Bogotá, Tercer Mundo/Facultad de Ciencias Humanas de la Universidad Nacional de Colombia, pp. 147-172.

Young, Kate (1988), "Reflections on Meeting Women's Needs", en Kate Young (ed.), Women and Economic Development: Local, Regional and National Planning Strategies, Oxford-Paris, Berg Publishers/UNESCO [traducción al español: Young, Kate (1991), "Reflexiones sobre cómo enfrentar las necesidades de las mujeres", en Guzmán et al., (eds.), Una nueva mirada: género en el desarrollo, Lima, Flora Tristán/Entre Mujeres].

(1997), "El potencial transformador en las necesidades prácticas: empoderamiento colectivo y el proceso de planificación", en Magdalena León (comp.), Poder y empoderamiento de las mujeres, Bogotá, Tercer Mundo/Facultad de Ciencias Humanas de la Universidad Nacional de Colombia, pp. 99-118. 